\title{
Prognostic analysis of esophageal cancer in elderly patients: metastatic lymph node ratio versus 2010 AJCC classification by lymph nodes
}

\author{
Ji-Feng Feng ${ }^{1,2}$, Ying Huang ${ }^{3}$, Lu Chen ${ }^{4}$ and Qiang Zhao ${ }^{1,2^{*}}$
}

\begin{abstract}
Background: Recent studies have proposed a new prognostic factor (metastatic lymph node ratio, or MLNR) for patients with esophageal cancer (EC). However, to the best of our knowledge, there have been no studies conducted to date regarding MLNR in elderly patients. The aim of this study was to determine the prognostic value of MLNR staging compared with the 2010 American Joint Committee on Cancer (AJCC) N staging in elderly patients with EC.

Methods: From January 2001 to December 2009, a retrospective analysis of 132 consecutive patients older than 70 years of age with esophageal squamous cell carcinoma (ESCC) was conducted. Prognostic factors for disease-specific survival were analyzed. Receiver operating characteristic curves were also plotted to verify the accuracy of MLNR staging and N staging for survival prediction.

Results: The disease-specific survival rates of N0, N1, N2 and N3 patients according to the AJCC Cancer Staging Manual Seventh Edition N staging were 65.5\%, 42.9\%, 22.2\% and 0, respectively (N0 vs N1, P = 0.017; N1 vsN2, = 0.050; N2 vs N3, $P<0.001$ ). The disease-specific survival rates of MLNRO, MLNR1, MLNR2 and MLNR3 patients were $65.5 \%, 45.0 \%, 21.1 \%$ and 0 , respectively (MLNRO vsMLNR1, $P=0.026$; MLNR1 vs MLNR2, $P=0.033$; MLNR2 vs MLNR3, $P=0.015$ ). The areas under the curve were 0.731 for the 2010 AJCC N staging and 0.737 for the MLNR staging.

Conclusion: MLNR is an independent predictor of survival in elderly patients with ESCC. MLNR staging predicts survival after EC similarly to the 2010 AJCC N classifications and should be considered an alternative to current N staging.
\end{abstract}

Keywords: Esophageal cancer, Lymph node ratio, Overall survival, TNM classification

\section{Background}

Esophageal cancer (EC) is the eighth most common type of cancer worldwide. In China, EC is the fourth most common cause of mortality, with 11 deaths per 100,000 in 2005 [1]. However, esophageal squamous cell carcinoma (ESCC) accounts for most EC cases in China, in contrast to the predominance of adenocarcinoma in the Western world [2]. Although advances have occurred in multidisciplinary treatment, surgical resection remains the treatment modality of choice. One of the main determinants of survival for patients undergoing esophagectomy is nodal

\footnotetext{
* Correspondence: Cdbzjzl@163.com

'Department of Thoracic Surgery, Zhejiang Cancer Hospital, No.38 Guangji Road, Banshan Bridge, Hangzhou 310022, China

${ }^{2}$ Key Laboratory Diagnosis and Treatment Technology on Thoracic Oncology, Hangzhou, Zhejiang Province 310022, China

Full list of author information is available at the end of the article
}

status. However, the best method of characterizing the extent of local lymph node metastases remains an area of controversy.

Recent studies have proposed a new prognostic factor (metastatic lymph node ratio, or MLNR) for EC patients [3-5]. Controversy exists concerning the optimal cutoff points for the MLNR to predict overall survival. The different study sizes, variable inclusion criteria and, most important, unreliable statistical methods used to determine the cutoff points between groups have contributed to this controversy. However, to the best of our knowledge, there have been no studies conducted to date regarding MLNR in elderly patients with EC.

The aim of this study was to determine the prognostic value of MLNR staging compared with the 2010 American

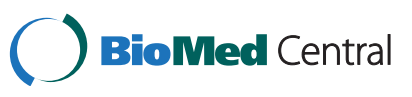




\begin{tabular}{|c|c|}
\hline Characteristics & Cases (n, \%) \\
\hline Age (mean $\pm S D, y r)$ & $73.6 \pm 2.6$ \\
\hline \multicolumn{2}{|l|}{ Gender } \\
\hline Female & $11(8.3)$ \\
\hline Male & $121(91.7)$ \\
\hline Tumor size (mean \pm SD, cm) & $4.6 \pm 1.7$ \\
\hline \multicolumn{2}{|l|}{ Tumor location } \\
\hline Upper & $6(4.5)$ \\
\hline Middle & $55(41.7)$ \\
\hline Lower & $71(53.8)$ \\
\hline \multicolumn{2}{|l|}{ Histologic grade } \\
\hline Well & $17(12.9)$ \\
\hline Moderately & $81(61.4)$ \\
\hline Poorly & $34(25.7)$ \\
\hline \multicolumn{2}{|l|}{ Tumor grade } \\
\hline $\mathrm{T} 1$ & $19(14.4)$ \\
\hline $\mathrm{T} 2$ & $16(12.1)$ \\
\hline T3 & 89 (67.4) \\
\hline T4 & $8(6.1)$ \\
\hline \multicolumn{2}{|l|}{ N stage } \\
\hline NO & $58(43.9)$ \\
\hline N1 & $42(31.8)$ \\
\hline N2 & $18(13.7)$ \\
\hline N3 & $14(10.6)$ \\
\hline TLN (mean $\pm S D, n)$ & $22.7 \pm 9.7$ \\
\hline NMLN (mean $\pm S D, n)$ & $2.2 \pm 3.7$ \\
\hline \multicolumn{2}{|l|}{ MLNR } \\
\hline MLNRO (0) & $58(43.9)$ \\
\hline MLNR1 $(>0, \leq 0.1)$ & $40(30.3)$ \\
\hline MLNR2 $(>0.1, \leq 0.3)$ & $19(14.4)$ \\
\hline MLNR3 (>0.3) & $15(11.4)$ \\
\hline
\end{tabular}

${ }^{a} E S C C$, esophageal squamous cell carcinoma; MLNR metastatic lymph node ratio; NMLN, number of metastatic lymph nodes; $T L N$, total lymph node.

Joint Committee on Cancer (AJCC) classification system by lymph nodes in elderly patients with ESCC.

\section{Methods}

\section{Patients}

We conducted a retrospective analysis of patients treated from January 2001 to December 2009. The sample population comprised 132 patients older than 70 years of age with ESCC who underwent curative esophagectomy in the Department of Thoracic Surgery, Zhejiang Cancer Hospital, Hangzhou, China. Patients who had received pre- and or postoperative chemotherapy and/or radiotherapy were excluded. We also excluded patients with
non-ESCC and gastroesophageal junction carcinoma, as well as patients who underwent surgical exploration without curative esophagectomy.

All of the above patients were followed up by posting letters or by telephone interviews. The last follow-up was on 30 November 2011. The clinicopathological and followup findings were collected and recorded in the database. All subjects gave their written informed consent to the study protocol, which was approved by the ethical committees of Zhejiang Cancer Hospital, Hangzhou, China.

\section{Surgery}

All patients were treated with radical resection. The standard surgical approach consisted of a limited thoracotomy on the right side and intrathoracic gastric reconstruction (the Ivor Lewis procedure) for lesions at the middle or lower third of the esophagus. Upper-third lesions were treated by cervical anastomosis (the McKeown procedure). In our institution, two types of lymphadenectomy were carried out as a standard procedure for ESCC. The majority of patients underwent two-field lymphadenectomy. In this cohort of patients, thoracoabdominal lymphadenectomy was performed, including the subcarinal, paraesophageal, pulmonary ligament, diaphragmatic and paracardial lymph nodes, as well as those located along the lesser gastric curvature, the origin of the left gastric artery, the celiac trunk, the common hepatic artery and the splenic artery. Three-field lymphadenectomy was performed only if the cervical lymph nodes were thought to be abnormal upon preoperative evaluation. All of the patients included in the study were restaged according to the classification system of the seventh edition of the American Joint Committee on Cancer (AJCC) Cancer Staging Manual (AJCC Seventh Edition) [6].

\section{Statistical analysis}

Statistical evaluation was conducted using SPSS version 17.0 software (SPSS Inc, Chicago, IL, USA). MLNR was defined as the ratio metastatic lymph nodes to total lymph nodes, and it was categorized into four groups $(0,>0, \leq 0.1,>0.1, \leq 0.3$ and $>0.3)$ as described in a previous study [4]. As this series described the prognosis of elderly patients with ESCC, a disease-specific survival analysis would more appropriately indicate the impact of the $\mathrm{N}$ classification system on cancer-related prognosis. The disease-specific survival was calculated by using the Kaplan-Meier method, and the difference was assessed by using the logrank test. Univariate and multivariate analyses of Cox regression proportional hazards model were performed to evaluate the prognostic parameters for survival. Spearman's correlation coefficient was calculated to assess the correlation related to MLNR. Receiver operating characteristic (ROC) curves were also plotted to verify the accuracy of MLNR staging and 2010 AJCC N staging 
for survival prediction. $P<0.05$ was considered to be statistically significant.

\section{Results}

\section{Patient characteristics}

The baseline characteristics are shown in Table 1 . Among the 132 patients, 11 (8.3\%) were women and 121 $(91.7 \%)$ were men. Their mean age was $73.7 \pm 2.6$ years, with an age range of 70 to 85 years. The most common tumor locations were the middle and lower esophagus (95.5\%). Total lymph node (TLN) harvest was highly variable (Figure 1A). A mean of $22.7 \pm 9.7$ nodes per patient was found during pathologic review (range: 6 to 61 nodes). The number of metastatic lymph nodes (NMLNs) per case ranged from 0 to 26 nodes (mean: $2.2 \pm 3.7$ nodes) (Figure 1B). The mean MLNR was 0.10 (range: 0 to 0.80$)$. Of the 132 patients, $58(43.9 \%)$ were classified as MLNR0, 40 (30.3\%) as MLNR1, 19 (14.4\%) as MLNR2 and 15 (11.4\%) as MLNR3.

\section{Prognostic factors}

Univariate analyses were performed to assess the predictive capability of each variable (Table 2). As expected, vessel involvement $(P=0.029)$, perineural invasion $(P=0.007)$, tumor grade $(=0.003), \mathrm{N}$ stage $(P<0.001)$ and MLNR stage $(P<0.001)$ were predictive of survival. There were no significant differences in terms of age, gender, tumor size or tumor location. Significant factors were extracted for further analysis, which was conducted by using the multivariate Cox proportional hazards model to adjust for the effects of covariates (Table 3). In that model, we demonstrated that tumor grade $(P=0.003), \mathrm{N}$ stage $(P<0.001)$ and MLNR stage $(P<0.001)$ were independent prognostic factors. However, better discrimination was found for the AJCC Nstage than MLNR classification in terms of hazard ratio (HR) (N2 vs N3).

\section{Disease-specific survival}

The disease-specific survival rate was $45.5 \%$. The survival curves developed according to the AJCC Seventh Edition $\mathrm{N}$ staging system, and the MLNR staging data are shown in Figure 2. The disease-specific survival rates of N0, N1, $\mathrm{N} 2$ and N3 patients according to AJCC Seventh Edition N staging were $65.5 \%, 42.9 \%, 22.2 \%$ and 0 , respectively (N0 vs N1, $P=0.017$; N1 vs N2, $P=0.050$; N2 vs N3, $P<0.001$ ). The disease-specific survival rates of MLNR0, MLNR1, MLNR2, and MLNR3 patients were 65.5\%, 45.0\%, 21.1\% and 0 , respectively (MLNR0 vs MLNR1, $P=0.026$; MLNR1 vs MLNR2, $P=0.033$; MLNR2 vs MLNR3, $P=0.015$ ). The survival rates were similar between patients with $\mathrm{N0}$ and N1 and those with MLNR0 and MLNR1, but the survival rates differed significantly between N1 vs N2 and MLNR1 vsMLNR2 $(P=0.050$ vs. $P=0.033)$.

\section{Correlation related to MLNR}

As expected, there was a positive correlation between the MLNR and NMLN $(r=0.914, P<0.001)$ (Figure 3A).
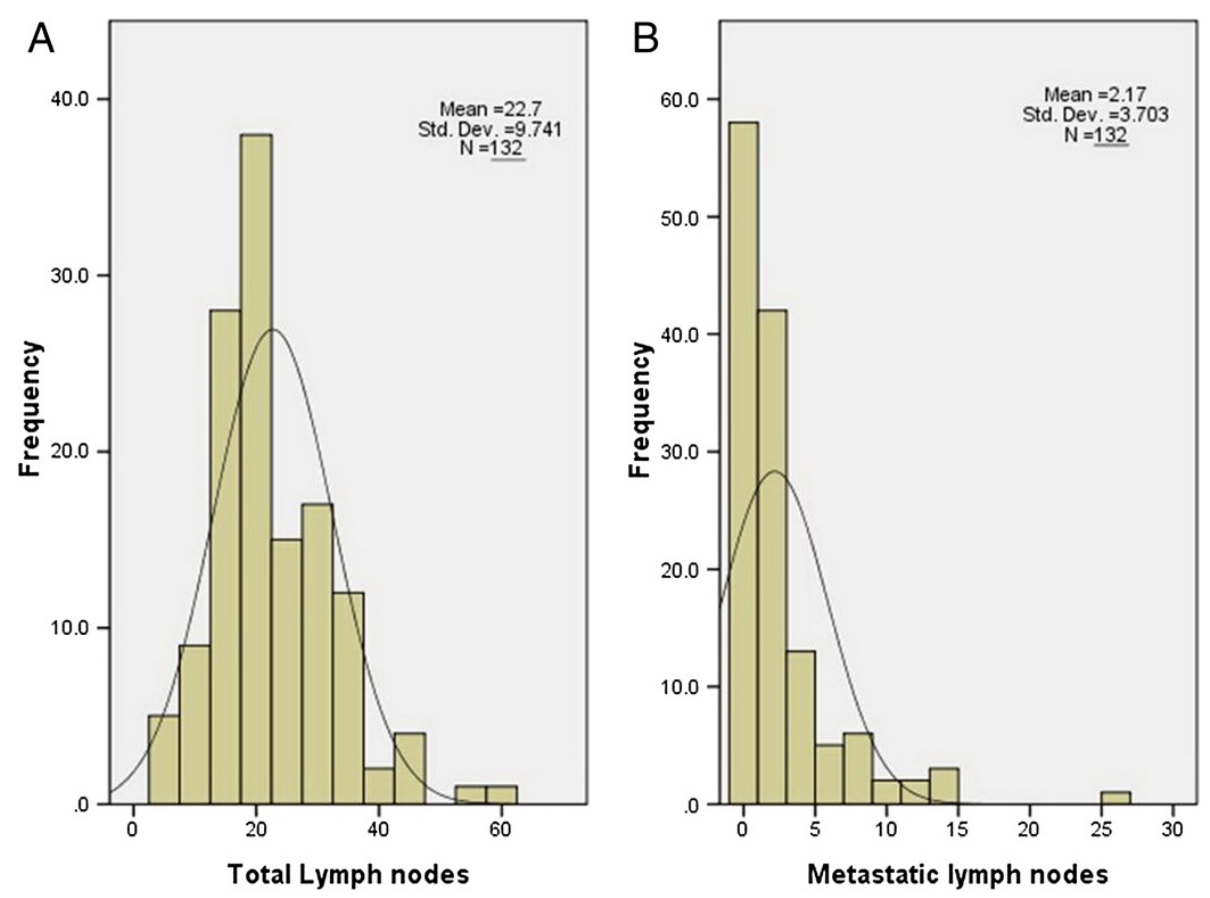

Figure 1 Histograms of the total (A) and metastatic (B) lymph nodes among surgery cohorts in ESCC patients. 
Table 2 Univariate Cox regression analysis of disease-specific survival ${ }^{\mathrm{a}}$

\begin{tabular}{|c|c|c|c|c|}
\hline Characteristics & Cases $(n, \%)$ & DSS (\%) & HR $(95 \% \mathrm{Cl})$ & $P$ value \\
\hline Age (yr) & & & & 0.838 \\
\hline$\leq 75$ & $97(73.5)$ & 46.4 & 1.000 & \\
\hline$>75$ & $35(26.5)$ & 42.9 & 1.055 (0.629 to 1.770$)$ & \\
\hline \multicolumn{5}{|l|}{ Gender } \\
\hline Female & $11(8.3)$ & 54.5 & 1.000 & 0.474 \\
\hline Male & $121(91.7)$ & 44.6 & 1.394 (0.561 to 3.460$)$ & \\
\hline Tumor size (cm) & & & & 0.081 \\
\hline$\leq 5$ & $91(68.9)$ & 52.7 & 1.000 & \\
\hline$>5$ & $41(31.1)$ & 29.3 & 1.524 (0.950 to 2.443$)$ & \\
\hline Tumor location & & & & 0.778 \\
\hline Upper & $6(4.5)$ & 50.0 & 1.000 & \\
\hline Middle & $55(41.7)$ & 45.5 & 0.938 (0.286 to 3.081$)$ & 0.916 \\
\hline Lower & $71(53.8)$ & 45.1 & 1.114 (0.344 to 3.613$)$ & 0.857 \\
\hline Histologic grade & & & & 0.073 \\
\hline Well & $17(12.9)$ & 58.8 & 1.000 & \\
\hline Moderately & $81(61.4)$ & 48.1 & 1.587 (0.712 to 3.538$)$ & 0.259 \\
\hline Poorly & $34(25.7)$ & 32.4 & 2.461 (1.053 to 5.752$)$ & 0.038 \\
\hline Vessel involvement & & & & 0.029 \\
\hline No & $100(75.8)$ & 48.0 & 1.000 & \\
\hline Yes & $32(24.2)$ & 37.5 & $1.786(1.061$ to 3.007$)$ & \\
\hline Perineural invasion & & & & 0.007 \\
\hline No & $113(85.6)$ & 49.6 & 1.000 & \\
\hline Yes & $19(14.4)$ & 21.1 & 2.198 (1.240 to 3.894$)$ & \\
\hline Tumor grade & & & & 0.003 \\
\hline $\mathrm{T} 1$ & $19(14.4)$ & 89.5 & & \\
\hline $\mathrm{T} 2$ & $16(12.1)$ & 68.8 & 4.319 (0.854 to 12.727$)$ & 0.069 \\
\hline T3 & $89(67.4)$ & 33.7 & 7.731 (2.868 to 14.844$)$ & 0.003 \\
\hline T4 & $8(6.1)$ & 25.0 & 10.551 (2.969 to 21.902) & 0.001 \\
\hline TLN (nodes) & & & & 0.729 \\
\hline$\leq 18$ & 47 (35.6) & 44.7 & 1.000 & \\
\hline$>18$ & $85(64.4)$ & 45.9 & 0.905 (0.597 to 1.427$)$ & \\
\hline N stage & & & & $<0.001$ \\
\hline No & $58(43.9)$ & 65.5 & 1.000 & \\
\hline N1 & $42(31.8)$ & 42.9 & 2.059 (1.136 to 3.732$)$ & 0.017 \\
\hline N2 & $18(13.7)$ & 22.2 & 4.122 (2.047 to 8.299$)$ & $<0.001$ \\
\hline N3 & $14(10.6)$ & 0 & 19.108 (8.503 to 42.939$)$ & $<0.001$ \\
\hline MLNR stage & & & & $<0.001$ \\
\hline MLNRO & $58(43.9)$ & 65.5 & & \\
\hline MLNR1 & $40(30.3)$ & 45.0 & 1.963 (1.070 to 3.602$)$ & 0.026 \\
\hline MLNR2 & $19(14.4)$ & 21.1 & 4.142 (2.902 to 8.202 ) & $<0.001$ \\
\hline MLNR3 & $15(11.4)$ & 0 & 12.037 (5.866 to 24.698$)$ & $<0.001$ \\
\hline
\end{tabular}

${ }^{a} \mathrm{Cl}$, confidence interval; $D S S$, disease-specific survival; $H R$, hazard ratio. 
Table 3 Multivariate Cox regression analysis of disease-specific survival $^{\mathrm{a}}$

\begin{tabular}{|c|c|c|}
\hline Characteristics & HR $(95 \% \mathrm{Cl})$ & $P$ value \\
\hline Vessel involvement & & 0.667 \\
\hline No & 1.000 & \\
\hline Yes & 0.868 (0.456 to 1.652 ) & \\
\hline Perineural invasion & & 0.205 \\
\hline No & 1.000 & \\
\hline Yes & 1.555 (0.786 to 3.075$)$ & \\
\hline Tumor grade & & 0.003 \\
\hline $\mathrm{T} 1$ & 1.000 & \\
\hline $\mathrm{T} 2$ & 2.177 (0.474 to 6.841$)$ & 0.198 \\
\hline $\mathrm{T} 3$ & 4.190 (0.733 to 10.148$)$ & 0.090 \\
\hline $\mathrm{T} 4$ & 7.170 (1.909 to 15.503$)$ & 0.010 \\
\hline N stage & & $<0.001$ \\
\hline No & 1.000 & \\
\hline N1 & 1.315 (0.709 to 2.440$)$ & 0.385 \\
\hline N2 & 2.366 (1.143 to 4.897$)$ & 0.020 \\
\hline N3 & 16.474 (6.390 to 42.468$)$ & $<0.001$ \\
\hline MLNR stage & & $<0.001$ \\
\hline MLNRO & 1.000 & \\
\hline MLNR1 & 1.173 (0.625 to 2.203$)$ & 0.619 \\
\hline MLNR2 & 2.678 (1.320 to 5.433$)$ & 0.006 \\
\hline MLNR3 & 7.860 (3.695 to 16.718$)$ & $<0.001$ \\
\hline
\end{tabular}

A negative correlation between MLNR and TLN, however, was not significant $(r=-0.140, P=0.110)$ (Figure 3B).

\section{ROC curve for disease-specific survival prediction}

The area under the curve (AUC) ratios were 0.731 (95\% CI: 0.647 to $0.816, P<0.001)$ for AJCC Seventh Edition $\mathrm{N}$ staging and 0.737 (95\% CI: 0.653 to $0.821, P<0.001$ ) for the MLNR staging, indicating that the MLNR staging was similar to the AJCC Seventh Edition N staging system and could be used as an alternative prognostic staging tool for ESCC patients (Figure 4).

\section{Discussion}

The aging of the population and a longer life expectancy have led to more elderly patients with cancers being referred for treatment. For many of them, in particular for those with EC, surgery remains the mainstay of treatment. There is no established cutoff to define a patient as "elderly" in relation to surgery, but most studies available to date have set the age limit at 70 years $[7,8]$. In our study, we determined the prognostic value of MLNR compared with $\mathrm{N}$ staging in EC patients older than 70 years of age. Our results suggest that MLNR might be a valuable prognostic factor of survival in elderly patients in EC. We conclude that MLNR staging predicts survival after EC similar to the AJCC Seventh Edition N staging classifications and should be considered as an alternative to current pathological $\mathrm{N}$ staging. To the best
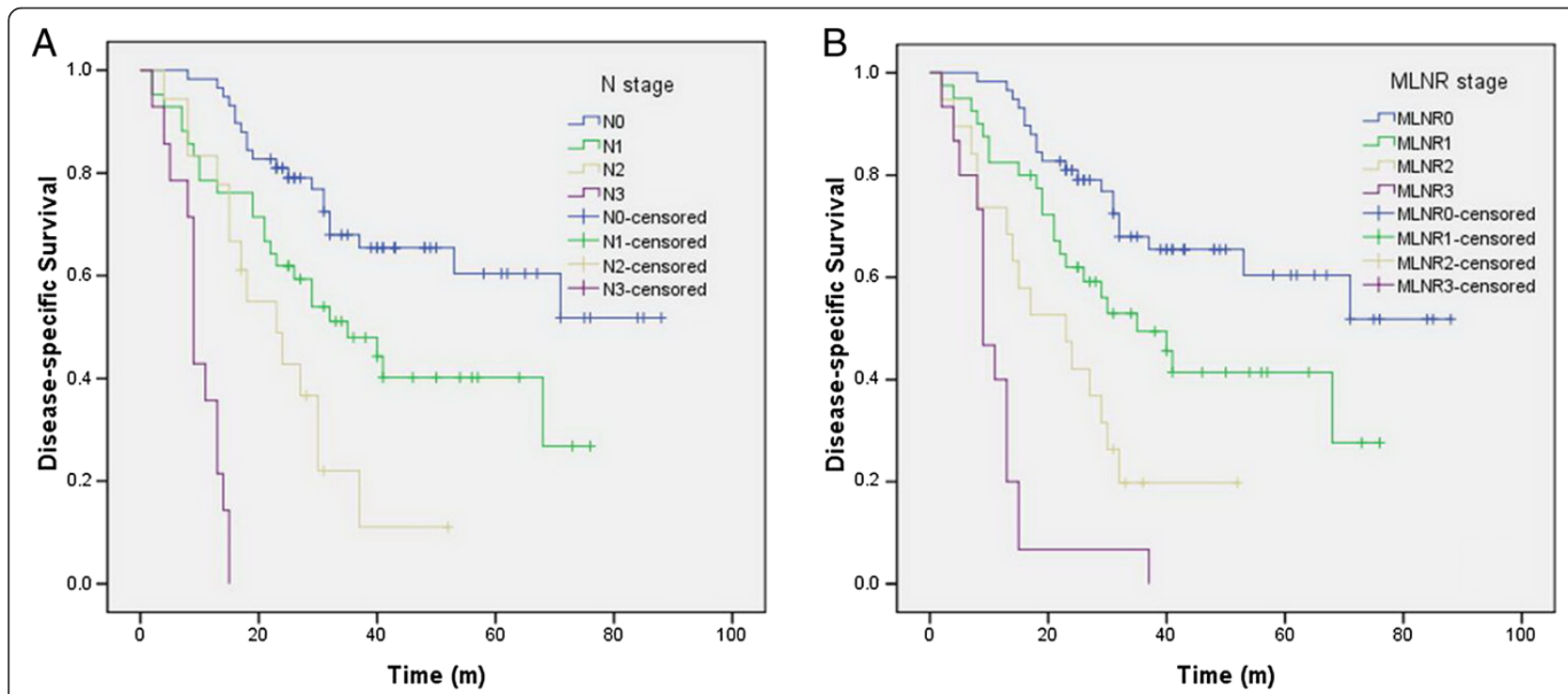

Figure 2 Impact of AJCC Seventh Edition N staging (A) and MLNR staging (B) on disease-specific survival of ESCC patients who underwent radical resection. (A) The disease-specific survival rates of N0, N1, N2 and N3 patients in AJCC Seventh Edition N staging were $65.5 \%, 42.9 \%, 22.2 \%$ and 0 , respectively (N0 vs N1, $P=0.017$; N1 vs N2, $P=0.050$; N2 vs N3, $P<0.001$ ). (B) The disease-specific survival rates of MLNRO, MLNR1, MLNR2 and MLNR3 patients were 65.5\%, 45.0\%, 21.1\% and 0, respectively (MLNRO vs. MLNR1, $P=0.026$; MLNR1 Vs MLNR2, $P=0.033 ;$ MLNR2 vs. MLNR3, $P=0.015)$. 

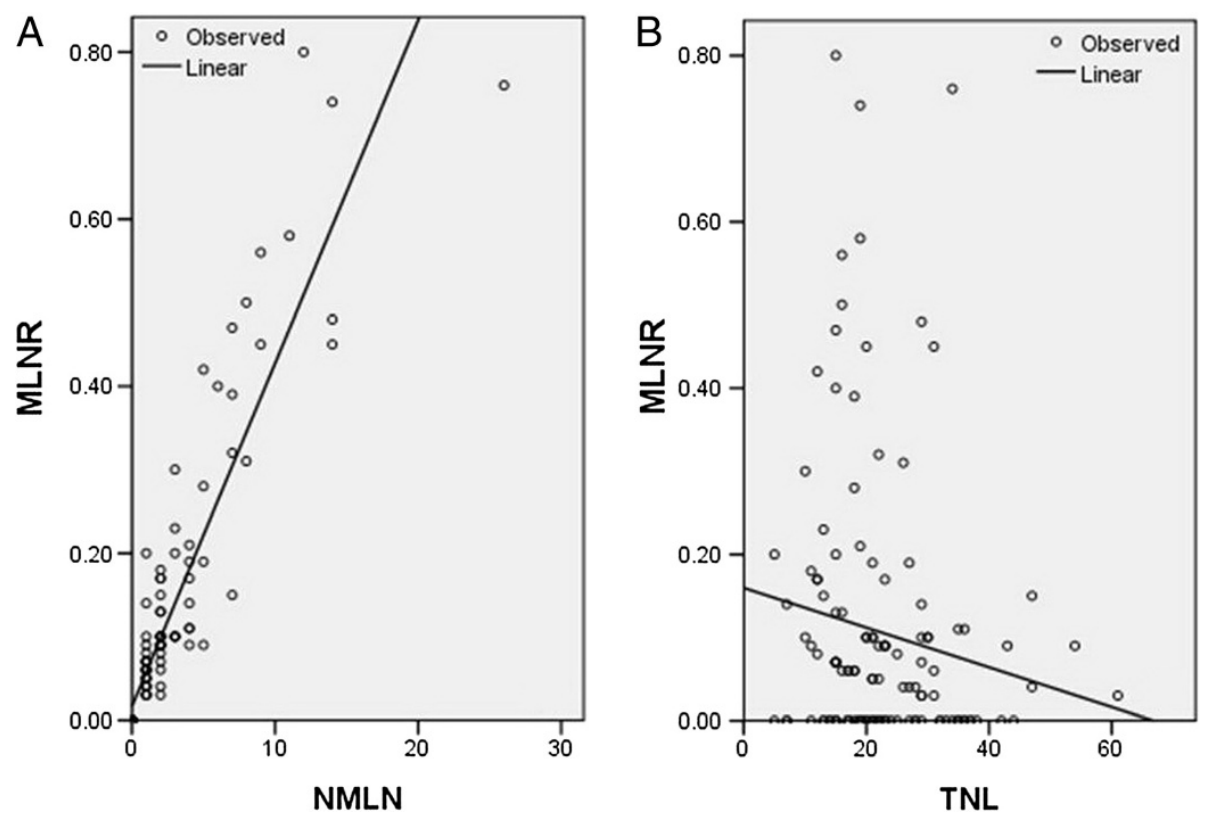

Figure 3 Correlation related to MLNR. (A) There was a positive correlation between the MLNR and NMLN $(r=0.914, P<0.001)$. (B) A negative correlation between MLNR and TLN, however, was not significant $(r=-0.140, P=0.110)$.

of our knowledge, our present studymay be the first to evaluate the value of MLNR vsAJCC Seventh EditionN staging in elderly patients with EC.

The ratio of metastatic to total lymph nodes (that is, the MLNR) has been shown to be a prognostic factor in $E C$, but the value of MLNR that is most predictive of

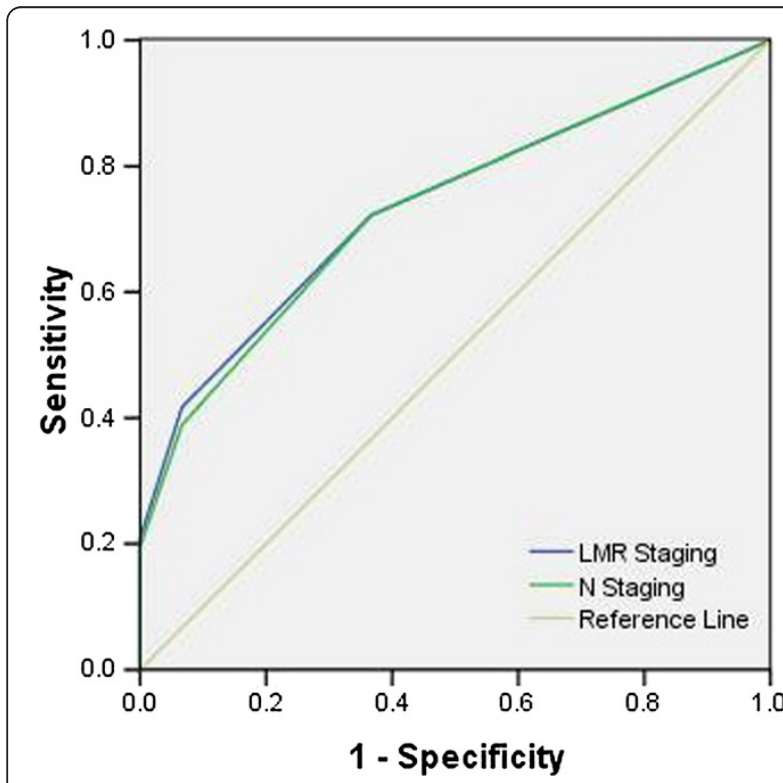

Figure 4 ROC curve for survival prediction. The ROC for MLNR staging is represented by the blue line with an $A \cup C=73.7 \%$, and the ROC for AJCC Seventh Edition N staging is represented by the green line with an $A \cup C=73.1 \%$. survival is being debated. Most of the studies published to date concerning the MLNR and survival have been based on the AJCC Sixth Edition classification system [3-5]. Furthermore, in terms of MLNR and survival, some studies have classified the MLNR into three groups, whereas other studies have used two classifications. In our study, the MLNR was categorized by deciles into 0 (MLNR0), $>0$ to $<0.1$ (MLNR1), 0.1 to $<0.3$ (MLNR2) and $\geq 0.3$ (MLNR3), based on the AJCC Seventh Edition classification system. We developed the MLNR intervals on the basis of our data to provide clinically relevant MLNR strata while probing to identify the subset of MLNR with the greatest predictive potential. In our study, the diseasespecific survival rates of MLNR0, MLNR1, MLNR2 and MLNR3 patients were $65.5 \%, 45.0 \%, 21.1 \%$ and 0 , respectively (MLNR0vs MLNR1, $P=0.026$; MLNR1 vs MLNR2, $P=0.033$; MLNR2 vs MLNR3, $P=0.015$ ). Wilson et al. [9] classified 144 patients into 4 groups according to MLNR: $0, \leq 25 \%,>25$ to $\leq 50 \%$ and $>50 \%$. Althoughan increasing MLNR was linearly associated with a worsening 5 -yr survival rate in their study, statistical significance was not achieved $(P=0.153)$. Bogoevski et al. [10] also classified 235 patients into four categories according to MLNR: $0,<11 \%, 11 \%$ to $33 \%$ and $>33 \%$, which is similar to our findings.

The question of how many lymph nodes should be dissected has been a point of debate in previous studies. Rizk et al. [11] reported that the prognosis of patients after esophagectomy worsens significantly after four or more lymph nodes have metastases, irrespective of $\mathrm{T}$ stage. 
Greenstein et al. [12] and Yang et al. [13] recommended 18 nodes as the minimum number of resectable lymph nodes, whereas Peyre et al. [14] recommended a minimum of 23 regional lymph nodes. Attendees at a consensus conference of experts in 1995 suggested that accurate pathological staging of EC requires resection of at least 15 nodes [15]. The International Union Against Cancer (UICC) and AJCC have proposed that at least sixlymph nodes should be removed during resection of EC. Hu et al. [16] used a cutoff of six removed lymph nodesas the definition of adequate nodal dissection. Their results showed that patients with six or more lymph nodes dissected had a higher rate of positive lymph nodes identified (46.9\% vs $30.3 \%$ ) and an improvement in overall survival that was statistically significant in pathologically node-negative patients. Accordingly, we excluded patients who had fewer than sixdissected lymph nodes (range: 6 to 61). In our study, we did not find any survival rate difference when using a cutoff of 18 nodes $(42.6 \%$ vs $44.7 \%, P=0.741)$.

In the present study, the correlation related to MLMR was determined. As expected, we found that there was a positive correlation between MLNR and NMLN $(r=0.914, P<0.001)$. There was a negative correlation between MLNR and TLN; however, the correlationwas not significant $(r=-0.140, P=0.110)$. ROC curves were plotted to verify the accuracy of MLNR staging and $\mathrm{N}$ staging for survival prediction. The AUCs were 0.731 for the 2010 AJCC N staging and 0.737 for the MLNR staging, indicating that the MLNR staging was similar to the AJCC Seventh Edition N staging system and could be used as an alternative prognostic staging tool for EC patients.

The potential limitations of the present study include the relatively small number of patients, the use of a retrospective analysis and the short duration of the mean follow-up. In addition, because the study used data from a single institution but with different pathologists and different surgeons, there may have been a lack of uniformity in measurement methods. Furthermore, owing to the limited number of patients in EC, our analysis may contain type I or type II errors. The results of the study should therefore be regarded with caution. Further studies are needed to explore its long-term effect.

\section{Conclusion}

In summary, our study suggests that the survival rate of elderly patients with ESCC can be categorized into four groups: MLNR0 (0), MLNR1 ( $>0$ to $\leq 0.1)$, MLNR2 $(>0.1$ to $\leq 0.3)$ and PLNR3 $(>0.3)$. We conclude that MLNR is an independent predictor of survival in elderly patients undergoing esophagectomy for EC. MLNR staging predicts survival after EC similarly to the 2010 AJCC N classifications and should be considered as an alternative to current pathological $\mathrm{N}$ staging.

\section{Competing interests}

The authors declare that they have no competing interests.

\section{Authors' contributions}

JFF conceived this study, collected data, performed analysis and drafted the manuscript. YH participated in the study design, literature search and study coordination. YH and LC performed data analysis and helped to draft the manuscript. QZ participated in the study design and helped to draft the manuscript. All authors read and approved the final manuscript.

\section{Acknowledgments}

None.

\section{Author details}

${ }^{1}$ Department of Thoracic Surgery, Zhejiang Cancer Hospital, No.38 Guangji Road, Banshan Bridge, Hangzhou 310022, China. ${ }^{2}$ Key Laboratory Diagnosis and Treatment Technology on Thoracic Oncology, Hangzhou, Zhejiang Province 310022, China. ${ }^{3}$ Department of Nursing, Zhejiang Cancer Hospital, Hangzhou, China. ${ }^{4}$ Department of Gynecologic Surgery, Zhejiang Cancer Hospital, Hangzhou, China.

Received: 26 December 2012 Accepted: 8 July 2013

Published: 18 July 2013

\section{References}

1. Zhao P, Dai M, Chen W, Li N: Cancer trends in China. Jpn J Clin Oncol 2010, 40:281-285.

2. Corley DA, Buffler PA: Oesophageal and gastric cardia adenocarcinomas: analysis of regional variation using the cancer incidence in five continents database. Int J Epidemiol 2001, 30:1415-1425.

3. Eloubeidi MA, Desmond R, Arguedas MR, Reed CE, Wilcox CM: Prognostic factors for the survival of patients with esophageal carcinoma in the U.S.: the importance of tumor length and lymph node status. Cancer 2002, 95:1434-1443.

4. Hou X, Wei JC, Xu Y, Luo RZ, Fu JH, Zhang $\sqcup$, Lin P, Yang HX: The positive lymph node ratio predicts long-term survival in patients with operable thoracic esophageal squamous cell carcinoma in China. Ann Surg Oncol 2013, 20:1653-1659.

5. Wijnhoven BP, Tran KT, Esterman A, Watson DI, Tilanus HW: An evaluation of prognostic factors and tumor staging of resected carcinoma of the esophagus. Ann Surg 2007, 245:717-725.

6. Rice TW, Rusch WW, Ishwaran H, Blackstone EH: Worldwide esophageal cancer collaboration: cancer of the esophagus and esophagogastric junction: data-driven staging for the seventh edition of the American joint committee on cancer/international union against cancer staging manuals. Cancer 2010, 116:3763-3773.

7. Poon R, Law SY, Chu KM, Branicki GJ, Wong J: Esophagectomy for carcinoma of the esophagus in the elderly. Ann Surg 1998, 227:357-364.

8. Ruol A, Portale G, Zaninotto G, Cagol M, Cavallin F, Castoro C, Sileni VC, Alfieri R, Rampado S, Ancona E: Results of esophagectomy for esophageal cancer in elderly patients: age has little influence on outcome and survival. J Thorac Cardiovasc Surg 2007, 133:1186-1192.

9. Wilson M, Rosato EL, Chojnacki KA, Chervoneva I, Kairys JC, Cohn HE, Rosato FE Sr, Berger AC: Prognostic significance of lymph node metastases and ratio in esophageal cancer. J Surg Res 2008, 146:11-15.

10. Bogoevski D, Onken F, Koenig A, Kaifi JT, Schurr P, Sauter G, Izbicki JR, Yekebas EF: Is it time for a new TNM classification in esophageal carcinoma? Ann Surg 2008, 247:633-641.

11. Rizk N, Venkatraman E, Park B, Flores R, Bains M, Rusch V: The prognostic importance of the number of involved lymph nodes in esophageal cancer: implications for revisions of the American joint committee on cancer staging system. J Thorac Cardiovasc Surg 2006, 132:1374-1381.

12. Greenstein AJ, Litle VR, Swanson SJ, Divino CM, Packer S, Wisnivesky JP: Effect of the number of lymph nodes sampled on postoperative survival of lymph node-negative esophageal cancer. Cancer 2008, 112:1239-1246.

13. Yang HX, Xu Y, Fu JH, Wang JY, Lin P, Rong TH: An evaluation of the number of lymph nodes examined and survival for node-negative esophageal carcinoma: data from China. Ann SurgOncol 2010, 17:1901-1911

14. Peyre CG, Hagen JA, DeMeester SR, Altorki NK, Ancona E, Griffin SM, Hölscher A, Lerut T, Law S, Rice TW, Ruol A, van Lanschot JJ, Wong J, 
DeMeester TR: The number of lymph nodes removed predicts survival in esophageal cancer: an international study on the impact of extent of surgical resection. Ann Surg 2008, 248:549-556.

15. Fumagalli U, Akiyama $\mathrm{H}$, DeMeester T: Resective surgery for cancer of the thoracic esophagus: results of a consensus conference held at the Vlth world congress of the international society for diseases of the esophagus. Dis Esophagus 1996, 9:30-38.

16. Hu Y, Hu C, Zhang H, Ping Y, Chen LQ: How does the number of resected lymph nodes influence TNM staging and prognosis for esophageal carcinoma? Ann Surg Oncol 2010, 17:784-790.

doi:10.1186/1477-7819-11-162

Cite this article as: Feng et al:: Prognostic analysis of esophageal cancer in elderly patients: metastatic lymph node ratio versus 2010 AJCC classification by lymph nodes. World Journal of Surgical Oncology 2013 11:162.

\section{Submit your next manuscript to BioMed Central and take full advantage of:}

- Convenient online submission

- Thorough peer review

- No space constraints or color figure charges

- Immediate publication on acceptance

- Inclusion in PubMed, CAS, Scopus and Google Scholar

- Research which is freely available for redistribution 